Bio-grafia Escritos sobre La Biología y su Enseñanza.

Edición Extra-Ordinaria. ISSN 2027-1034 P. p387-394

Memorias del VII Encuentro Nacional de Experiencias en la Enseñanza de la

Biología y la Educación Ambiental y II Congreso Nacional de Investigación en la Enseñanza de la Biología

\title{
DISEÑO DE UNA UNIDAD DIDACTICA PARA LA ENSEÑANZA DE LA IRRITABILIDAD EN LOS SERES VIVOS DESDE UNA VISION SISTEMICA
}

\section{DESIGN DIDACTIC UNIT FOR THE EDUCATION OF THE IRRITABILITY IN THE ALIVE BEINGS FROM A SYSTEMIC VISION}

\author{
Lizeth Ariza Bareño ${ }^{1}$ \\ Zolangie González Molina \\ Paola Sánchez Herrera ${ }^{3}$
}

\section{Resumen}

Se presenta la propuesta del diseño de una unidad didáctica que se realizó en el eje dinámica y mantenimiento de los sistemas de la Licenciatura en Biología de la Universidad Pedagógica Nacional, donde se planteó como objetivo diseñar una unidad didáctica para la enseñanza de la irritabilidad en los seres vivos a través de los diseños experimentales para estudiantes de grado séptimo del Colegio Guillermo Cano Isaza (Bogotá-Colombia). Donde para la elaboración de las actividades se tuvo en cuenta a Sanmartí (2000), quien propone que partiendo del contexto las actividades se deben dividir en actividades de iniciación, actividades de desarrollo y actividades de finalización. Por ultimo encontramos que la unidad didáctica es un implemento importante dentro de los procesos metacognitivos que desempeña el maestro, puesto que posibilita que este muestre sus habilidades al momento de plantear elementos investigativos que sean de gran aporte para su formación profesional.

Palabras Claves: Unidad Didáctica, Irritabilidad, Visión sistémica, Diseños experimentales.

\section{Abstract}

We present the proposed design of a unit that was made in the shaft dynamics and maintenance of systems in Biology of the National Pedagogical University, where he set a goal to design a teaching unit for teaching irritability in living through experimental designs for seventh grade students of the College Guillermo Cano Isaza (Bogota-Colombia). Where to prepare activities was considered to Sanmartí

\footnotetext{
${ }^{1}$ Universidad Pedagógica Nacional. Estudiante de Licenciatura en Biología Lisa19111@hotmail.com

2 Universidad Pedagógica Nacional. Estudiante de Licenciatura en Biología Zolec14@hotmail.com

${ }^{3}$ Universidad Pedagógica Nacional. Estudiante de Licenciatura en Biología Neop 458@hotmail.com
} 
Bio-grafia Escritos sobre La Biología y su Enseñanza.

Edición Extra-Ordinaria. ISSN 2027-1034 P. p387-394

Memorias del VII Encuentro Nacional de Experiencias en la Enseñanza de la

Biología y la Educación Ambiental y II Congreso Nacional de Investigación en la Enseñanza de la Biología

(2000), who proposed that the activities from the context should be divided into initiation activities, development activities and completion activities. Finally we find that an implement teaching unit is important in metacognitive processes performed by the teacher, since this show enables their abilities when framing research elements that are of great contribution to their professional training.

Keywords: Didactic Unit, Irritability, systemic vision, experimental designs.

\section{Introducción}

Dentro del PCLB (Proyecto Curricular de Licenciatura en Biología) se hace pertinente la construcción de proyectos semestrales que aporten al desarrollo pedagógico y disciplinar del estudiante, y a su vez al enfoque que tiene la Universidad Pedagógica Nacional como formadora de maestros, la cual está enmarcada en la búsqueda de la pedagogía y la didáctica en el contexto educativo. Estos proyectos surgen a partir de los intereses de los estudiantes y los aportes de los maestros, quienes se encargan de guiar un proceso formativo para la construcción de los mismos. En cada eje curricular se busca responder a un NIP (núcleo integrador de problemas), el cual muestra las necesidades académicas de dicho eje, por medio de una pregunta problema, la cual busca ser respondida por todos los componentes del semestre; para el caso particular del eje dinámica y mantenimiento de los sistemas, se plantea el interrogante ¿Es posible la autorregulación de los sistemas?

En este sentido, para hallar una posible respuesta al NIP, se planteó la elaboración de una unidad didáctica, en la cual se muestra el aprendizaje del patrón irritabilidad en los seres vivos a partir de una visión sistémica, la cual tiene en cuenta las dinámicas sociales y culturales de un grupo en donde surgen diferentes problemáticas alrededor de dicho proceso; así mismo, se toman elementos de los diferentes sistemas como lo son el social, económico, cultural, político, y los que están relacionados con los maestros, el sistema educativo, pedagógico e investigativo, hallando una red de relaciones donde la visión sistémica desempeña un papel indispensable en la enseñanza del patrón irritabilidad como eje central y temático.

Esta unidad didáctica se propone para estudiantes de grado séptimo del colegio Guillermo Cano Isaza, el cual, se ubica en el barrio México, en la localidad de Ciudad Bolívar, viéndonos en la necesidad de brindar elementos que aporten al 
Bio-grafia Escritos sobre La Biología y su Enseñanza.

Edición Extra-Ordinaria. ISSN 2027-1034 P. p387-394

Memorias del VII Encuentro Nacional de Experiencias en la Enseñanza de la

Biología y la Educación Ambiental y II Congreso Nacional de Investigación en la Enseñanza de la Biología

aprendizaje de este patrón en un contexto culturalmente diverso, que tiene un origen y riqueza cultural bastante amplia, mostrando así un centro de diferentes concepciones, visiones de mundo y formas de actuar, según su referente geográfico enmarcado en aspectos históricos, culturales y sociales.

Es necesario mostrar que esta población, aunque no se percate de ello, se encuentra susceptible al patrón de irritabilidad, puesto que a su alrededor se observa la presencia de diferentes problemáticas ambientales como el rio Tunjuelito, las ladrilleras, la extracción de sementeras, que afectan directamente a su medio interno y externo, provocando así, respuestas negativas ante diferentes estímulos. Como tal, la comunidad educativa no está ejerciendo acciones concretas para contrarrestar el daño que se está generando a nivel ambiental, ya que es evidente el deterioro paisajístico, la deforestación, las curtiembres, las practicas inadecuadas de extracción y formación minera, lo cual genera un impacto considerable a nivel de salubridad, no solo de la población como tal, sino también de la localidad.

Por lo anterior, se pretende generar una reflexión a partir del patrón irritabilidad en los estudiantes, para que así ellos transformen comportamientos que les afecten y afecten al entorno, y a su vez, generar actitudes investigativas y analíticas por medio de ejercicios como la observación, interpretación y argumentación, todo esto apoyado en los estándares curriculares para Ciencias Naturales y Educación ambiental (2004) donde los estudiantes de grado séptimo deben tener unos conocimientos de procesos biológicos básicos, haciéndose presente la irritabilidad como un patrón de organización y proceso vital en los seres vivos.

Es por esto que la realización de esta unidad didáctica busca reconocer la irritabilidad como un patrón de organización que caracteriza a los seres vivos con relación a su entorno, teniendo en cuenta una mirada sistémica del mundo de lo vivo permitiendo ver a éste como un sistema auto-regulado, un trabajo pertinente dentro del contexto anteriormente mencionado, si tenemos en cuenta las problemáticas en las que se encuentra inmersa la población, objeto de nuestro estudio.

Como objetivo general se pretende diseñar una unidad didáctica para la enseñanza de la irritabilidad en los seres vivos a través de los diseños experimentales para estudiantes de grado séptimo del Colegio Guillermo Cano 
Bio-grafia Escritos sobre La Biología y su Enseñanza.

Edición Extra-Ordinaria. ISSN 2027-1034 P. p387-394

Memorias del VII Encuentro Nacional de Experiencias en la Enseñanza de la

Biología y la Educación Ambiental y II Congreso Nacional de Investigación en la Enseñanza de la Biología

Isaza (Bogotá-Colombia). Así mismo, se busca llegar a consolidar este objetivo por medio de objetivos específicos tales como:

- Generar actitudes investigativas y analíticas para la comprensión del concepto irritabilidad en los sistemas vivos por medio de ejercicios educativos.

- Entender que los seres vivos reaccionan ante estímulos del ambiente, por medio de diseños experimentales que permitan evidenciar la irritabilidad.

- Suscitar aprendizajes significativos en los estudiantes de grado séptimo del Colegio Guillermo Cano Isaza (Bogotá-Colombia) sobre el patrón irritabilidad en los seres vivos.

La realización de esta unidad didáctica permite una articulación entre conceptos biológicos, desde una perspectiva sistémica, aportando a la construcción de conocimiento desde el contexto, adecuando así una temática necesaria y pertinente, teniendo en cuenta las necesidades del mismo.

\section{Metodología}

La unidad didáctica esta propuesta para estudiantes de grado séptimo de La institución Educativa Distrital Guillermo Cano Isaza ubicada en el Barrio México de la localidad de Ciudad Bolívar (Bogotá, Colombia), bajo un enfoque constructivista piagetano y desde la perspectiva de Ausubel del aprendizaje significativo, donde la 'enseñanza' no se refiere a una simple transmisión de conocimientos (concepcion tradicional), en cambio, se refiere a un intercambio de información entre profesor y estudiante, como lo dice Ausubel (SF) no sólo se trata de saber la cantidad de información que posee, sino cuales son los conceptos y proposiciones que maneja, posibilitando la construcción de entidades conceptuales mucho mas organizadas, que una acumulacion memoristica de datos, dentro de la actividad cognoscitiva de los estudiantes; esto es una genuina construcción del conocimiento.

Para el desarrollo de la Unidad Didáctica se plantea la elaboración que conforman la unidad didáctica puede ser variada, en este caso tuvimos en cuenta a Sanmartí (2000), donde este autor nos propone que partiendo del contexto podemos tener una guía para la construcción de estas actividades las cuales se dividen en tres partes que son: 
Bio-grafía Escritos sobre la Biología y su Enseñanza.

Edición Extra-Ordinaria. ISSN 2027-1034 P. p387-394

Memorias del VII Encuentro Nacional de Experiencias en la Enseñanza de la

Biología y la Educación Ambiental y II Congreso Nacional de Investigación en la Enseñanza de la Biología

- Actividades de iniciación: Indagación de ideas previas.

- Actividades de desarrollo: Diseños experimentales.

- Actividades de finalización y acabado: Evaluación.

\section{Resultados Y Discusión}

El resultado obtenido en este trabajo es unidad didáctica que responde al patrón irritabilidad, siendo este abordado por medio de la pregunta ¿Cómo responden los seres vivos ante los estímulos del entorno? En esta unidad se pretende responder a la pregunta inicial desde las perspectivas a nivel de microorganismos, plantas y animales. Es por esto que se realiza una caracterización acerca del diseño de la Unidad didáctica y de las actividades que allí se plantean para el desarrollo del concepto Irritabilidad en los seres vivos.

\section{Caracterización del diseño de la unidad didáctica}

Dentro de la formación como futuros licenciados en Biología, el profesor en los procesos de enseñanza, realiza unas transformaciones de saberes específicos de una disciplina (en nuestro caso, disciplina científica), esta 'transformación' es denominada 'transposición didáctica' donde, según Chevallard (1991) se realiza el trabajo que transforma un objeto de saber a enseñar, en un objeto de enseñanza.

Esta unidad didáctica se realiza acerca de la irritabilidad en los sistemas vivientes, - microorganismos, plantas y animales -, dónde se quiere que los estudiantes reconozcan a esta como un patrón de organización que caracteriza a los seres vivos.

Teniendo en cuenta lo anterior, estamos afirmando entonces que la enseñanza es un eje central de la Pedagogía, y que se requiere de un discurso metódico que incluya la forma de 'ser' y 'hacer' del profesor, los procedimientos para enseñar ciertos contenidos, un conocimiento-objeto de enseñanza (esto es, de la disciplina a enseñar), condiciones o estrategias bajo las cuales debe ser enseñado un saber especifico y lo que caracteriza a la forma de conocer y aprender. Ese discurso que busca tal relación, es lo que conforma lo que denominamos 'didáctica'; la didáctica es una disciplina teórica que se ocupa de estudiar la acción pedagógica, es decir, las prácticas de la enseñanza, y que tiene como misión describirlas, explicarlas, fundamentar y enunciar normas para la mejor resolución de los problemas que estas prácticas plantean a los profesores. (Camilloni, 2007). 
Bio-grafía Escritos sobre la Biologia y su Enseñanza.

Edición Extra-Ordinaria. ISSN 2027-1034 P. p387-394

Memorias del VII Encuentro Nacional de Experiencias en la Enseñanza de la

Biología y la Educación Ambiental y II Congreso Nacional de Investigación en la

\section{Enseñanza de la Biología}

La preparación de la unidad didáctica conlleva la elección de los contenidos científicos (en nuestro caso, acerca de la irritabilidad), la organización y secuencia de los mismos, el diseño de actividades de clase y de posibles tareas extraescolares, la anticipación de las dificultades que pueden encontrar los alumnos, etc. (Campanario, 1999).

Teniendo en cuenta a Campanario, tomamos como referencia la elección de los contenidos establecidos en los estándares curriculares para Ciencias Naturales y Educación Ambiental (2004) donde encontramos que los estudiantes de séptimo grado deben tener unos conocimientos de procesos biológicos básicos donde, en este caso, trabajaremos los procesos vitales y organización de los seres vivos.

Para ello se plantean unos objetivos en la unidad didáctica que corresponde a la irritabilidad como un patrón de organización en los sistemas vivos; posteriormente, se realiza una actividad para dar cuenta desde la perspectiva constructivista, las ideas previas que tiene los estudiantes para partir de ellas, y construir un conocimiento escolar que posibilite generar un aprendizaje significativo, el cual, según Ausubel (1976) es dónde el estudiante relaciona de manera no arbitraria y sustancial la nueva información de los contenidos, experiencias previas y familiares que ya posee en su estructura cognitiva.

\section{Caracterización de actividades}

Luego de tener en cuenta las ideas previas, se plantea una breve introducción pensada en los estudiantes, dónde se nombra que los seres vivos poseen características generales y específicas como la irritabilidad que les permite tener una organización en el sistema viviente.

Teniendo en cuenta los objetivos se plantea una explicación breve acerca de que seres vivos responden a esta característica, los cuales son microorganismos, plantas y animales, donde se dice que, dependiendo del estímulo, va a ver una respuesta y un receptor especifico, lo cual, permite reconocer los cambios físicos y químicos.

Se recuerda un poco sobre cada ser vivo, para este caso concreto, se inicia con los microorganismos ya que se trata de dar a la vez, una noción de sucesión de lo vivo, en la cual, lo primero que se genera son estos organismos seguido de las plantas y por último los animales, según las características evolutivas que dieron origen a la vida, presentando un pequeño y general resumen de cada ser vivo, 
Bio-grafía Escritos sobre la Biologia y su Enseñanza.

Edición Extra-Ordinaria. ISSN 2027-1034 P. p387-394

Memorias del VII Encuentro Nacional de Experiencias en la Enseñanza de la

Biología y la Educación Ambiental y II Congreso Nacional de Investigación en la Enseñanza de la Biología

ilustrando esas características generales y a la vez mostrando como cada organismo responde a la irritabilidad por medio de los estímulos generados por el entorno.

En cada actividad se hace énfasis en la caracterización de los estímulos y respuestas que presentan los diferentes organismos por medio de cuadros en los que se explica e ilustra dicha información. De igual forma, en estas actividades se plantean unos diseños experimentales con la finalidad de generar en el estudiante una reflexión acerca de los estímulos que inciden en los organismos y como el entorno afecta el desarrollo y sostenimiento de los mismos. Además se quiere que los estudiantes a través de estos diseños experimentales evidencien los problemas en cuanto a la irritabilidad que se encuentran en su contexto escolar. Los diseños experimentales están planteados para que los estudiantes identifiquen las principales características de la irritabilidad en los sistemas vivientes y los relacionen con los problemas de su entorno y contexto escolar, por esto los materiales y recursos necesarios para el desarrollo de estos son objetos y organismos que se encuentran fácilmente en el entorno del estudiante.

\section{Conclusiones}

- La implementación de diseños experimentales dentro de la escuela, fomentan el aprendizaje significativo de los estudiantes, a partir de la experimentación de los procesos que se llevan a cabo, tanto en los sistemas vivientes, como en su entorno.

- La unidad didáctica es un implemento importante dentro de los procesos metacognitivos que desempeña el maestro, puesto que posibilita que este muestre sus habilidades al momento de plantear elementos investigativos que sean de gran aporte para su formación profesional.

- La integración de los conocimientos disciplinares y pedagógicos permiten que el maestro se forme integralmente, articulando de manera asertiva la comprensión de sus conocimientos a partir de la reflexión en su ejercicio pedagógico.

- la irritabilidad se puede tomar como patrón que afecta al medio creando en ocasiones desequilibrios biológicos; de acuerdo con esto, es pertinente 
Bio-grafía Escritos sobre La Biología y su Enseñanza.

Edición Extra-Ordinaria. ISSN 2027-1034 P. p387-394

Memorias del VII Encuentro Nacional de Experiencias en la Enseñanza de la Biología y la Educación Ambiental y II Congreso Nacional de Investigación en la Enseñanza de la Biología

crear una conciencia planetaria que busque mejorar la calidad de vida de la población en general, con la implementación de estrategias pedagógicas que sean de gran aporte para la comunidad educativa.

\section{Bibliografía}

ADÚRIZ-BRAVO A., E IZQUIERDO-AYMERICH M. (2002). Acerca de la didáctica de las ciencias como disciplina autónoma. Revista Electrónica de Enseñanza de las Ciencias, Vol. 1, № 3, 130-140.

AUSUBEL, D.(1976). Teoría del aprendizaje significativo. Pp 1-10.informacion recuperada el día 15 de marzo del 2010 de a.com.ar/docentes/tuarticulo/educacion/ausubel/index.html

CAMILLONI A., Cols E., Basabe L. y Feeney S. (2007). El saber didáctico. Editorial Paidós. $1^{a}$ ed., Buenos Aires. Argentina.

CAMPANARIO, J. M. y MOYA, A. 1999. ¿Cómo enseñar ciencias? Principales Tendencias y propuestas. En: Revista Enseñanza de las Ciencias. Pp 179 - 192.

CAPRA, FRITJOF (1999) la trama de la vida. Una nueva perspectiva de los seres vivos. Editorial Anagrama.

CHEVALLARD, Y (1991).La Transposición Didáctica: Del saber sabio al saber Enseñado. Ed. Aique, Buenos Aires.

ESTÁNDARES BÁSICOS DE CIENCIAS NATURALES. (2004). Formar en ciencias el desafío: lo que necesitamos saber y saber hacer. Ministerio de Educación Nacional. Colombia

SANMARTÍ, N. (2000). El diseño de unidades didácticas. En F.J. Perales y. P. Cañal (Eds.), Didáctica de las ciencias experimentales (pp. 239-266) 